\title{
RÉPONSE ADAPTATIVE AUX RADIATIONS IONISANTES DES CELLULES HUMAINES
}

\author{
O. RIGAUD
}

\author{
Commissariat à l'Energie Atomique \\ Institut de Protection et de Sureté Nucléaire, \\ DPHD/SARAM, BP. 6, 92265 Fontenay aux Roses cedex
}

La réponse adaptative apparait lorsque des cellules sont exposées à une dose faible d'irradiation n'induisant pas de dommages détectables (de l'ordre du cGy ou moins) puis quelques heures plus tard traitées par une dose forte (plusieurs Gy); les cellules ont alors une sensibilité réduite aux effets génotoxiques de la dose forte.

Cette réponse radio-adaptative a été décrite pour la première fois en 1984 par Olivieri et al. (1): la pré-exposition de lymphocytes humains à une faible dose d'irradiation rend les cellules réfractaires à l'effet clastogène induit par la dose forte administrée ultérieurement; l'adaptation résulte en une réduction de $50 \%$ du taux d' aberrations chromatidiques attendu pour la forte dose. Cette réponse radio-adaptative à l'effet clastogène a fait l'objet de nombreuses publications et certaines caractéristiques de ce phénomène sont bien définies (2-5). Elle peut être induite in vitro dans les cellules de rongeurs, de plantes et aussi in vivo chez la souris (5). Une certaine variabilité de la réponse adaptative existe au sein d'une population d'individus normaux (6).

La réduction de l'effet clastogène a lieu dans les 4-6 heures suivant l'exposition à la dose adaptative et dépend de la synthèse de novo de protéines. La réponse adaptative peut être abolie par la 3-aminobenzamide, suggérant l'induction par la faible dose d'un système de réparation responsable de la diminution des aberrations chromatidiques dans les cellules adaptées (7-8).

Il a été montré que les lymphocytes humains radio-adaptés sont moins sensibles à l'effet inducteur de mutations géniques par les rayonnements ionisants (9-10).

Nous avons montré récemment que la fréquence de mutants HPRT- est réduite dans des cellules issues d'une lignée lymphoblastoide humaine préexposées à une faible dose d'irradiation (11). De plus, la nature moléculaire des mutations radio-induites au locus HPRT a été déterminée par la méthode de Southern. 
Le gène HPRT, gène non essentiel, offre l'avantage de pouvoir détecter un large spectre de mutations allant de la mutation ponctuelle à la délétion totale dugène. Les mutations induites au locus HPRT dans les cellules irradiées par une dose forte unique sont majoritairement de type délétion (12). Notre travail avait pour but de rechercher si la distribution des types moléculaires de mutations était modifiée selon que les cellules aient été ou non pré-exposées à une faible dose adaptative, ceci afin de comprendre le mécanisme cellulaire impliqué dans l'adaptation à l'effet mutagène.

Effets cytotoxique et mutagène de l'irradiation dans les cellules lymphoblastoides humaines

Le traitement adaptatif consiste en une pré-exposition des cellules à une dose de 0,02 Gy suivie d'une forte dose d'irradiation de 4 Gy (source ${ }^{137} \mathrm{Cs}$ ) administrée 6 heures après. La survie et la fréquence des mutants au locus HPRT ont été mesurées par la technique de clonage des cellules en dilution limite après les deux conditions de traitement.

La survie est similaire que les cellules aient été irradiées avec 4 Gy en dose unique $(8,7 \%$ de survie) ou donnée 6 heures après la faible dose $(10 \%$ de survie). Par contre, comparée à une irradiation unique de $4 \mathrm{~Gy}$, le traitement $0,02 \mathrm{~Gy}+4 \mathrm{~Gy}$ induit une réduction de $70 \%$ de la fréquence de mutation HPRT $^{-}\left(24,4\right.$ vs 70,7 mutants / $10^{6}$ cellules survivantes ).

La pré-exposition des cellules $\mathrm{AHH}-1$ à une faible dose de 0, 02 Gy est donc capable de réduire la sensibilité des cellules à l'effet mutagène de la forte dose, alors que la survie n'est pas modifiée. Ceci suggère que les lésions léthales induites par l'irradiation ou/et leur réparation diffèrent de celles conduisant aux mutations.

Nature moléculaire des mutations induites par lirradiation dans les cellules adaptées ou non par une faible dose.

Nos résultats suggèrent l'induction par la faible dose d'un mécanisme cellulaire responsable de la réduction de lésions prémutagènes. L'analyse moléculaire des mutations induites par les deux traitements soit adaptatif soit en dose unique devrait permettre de savoir si ce mécanime agit sur un certain type de lésions prémutagènes.

A partir des cultures de cellules traitées avec 0 Gy, 0, 02 Gy, 4 Gy et 0, 02 Gy + 4 Gy, 94 mutants ont été isolés de façon indépendante. L'ADN de chaque mutant a été analysé par la méthode de Southern après digestion par les 
deux enzymes de restriction EcoRI et PstI séparément puis hybridation à une sonde d'ADNc du gène HPRT humain.

Dans le tableau ci-dessous sont résumés les résultats obtenus:

$\begin{array}{ccl}\text { traitement mutants } & \begin{array}{l}\text { mutants non } \\ \text { anałysés }\end{array} \\ & \text { réarrangés }\end{array}$

mutants

0

24

$17(71 \%)$

$1(4 \%)$

$6(25 \%)$

$7(29 \%)$

$0,02 \mathrm{~Gy}$

17

$10(59 \%)$

$2(12 \%)$

$5(29 \%)$

$7(41 \%)$

4 Gy

27

$6(22 \%)$

$726 \%)$

$14(52 \%)$

$21(78 \%)$

0.02 Gy+4Gy 26

$15(58 \%)$

$6(23 \%)$

$5(19 \%)$

$11(42 \%)$

La majorité des mutants spontanés ou induits par la faible dose de 0, 02 Gy ne présentent pas de réarrangements visibles du gène HPRT ( $71 \%$ et $59 \%$ des mutants analysés respectivement).

Parmi les mutants induits par la dose unique de 4 Gy, 78\% (21/27) ont des altérations détectables du gène HPRT (mutants réarrangés). Par contre, dans les conditions de traitement radio-adaptatif, seulement $42 \%(15 / 26)$ de mutants réarrangés sont induits, soit une réduction significative comparés à ceux induits par une dose unique $(\mathrm{p}=0,02 \%)$. De plus parmi ces derniers, la fraction de mutants présentant des délétions partielles du gène est préférentiellement réduite $(19 \%$, soit une diminution significative $(p=$ $0,03 \%$ ) comparée aux $52 \%$ de mutants partiellement délétés induits par la dose unique de $4 \mathrm{~Gy}$ ).

En conclusion, ces résultats montrent des changements du spectre moléculaire de mutation selon que les cellules aient été soumises à une préexposition à une faible dose: la dose adaptative semble agir préférentiellement sur les lésions prémutationnelles aboutissant à des délétions.

En analogie avec les résultats sur la réponse adaptative contre l'effet clastogène indiquant l'induction par la faible dose d'un système de réparation, nous suggérons que la réduction de l'effet mutagène par la dose forte de 4 Gy sur les cellules pré-exposées à une faible dose pourrait être due à l'induction d'un système de réparation fidèle. Ce système semblerait agir 
préférentiellement sur la classe des lésions prémutagènes qui aboutissent à des mutations de type délétion.

\section{BIBLIOGRAPHIE}

1. OLIVIERI G., BODYCOTE J. AND S. WOLFF (1984) Adaptive response to low concentration of radioactive thymidine. Science, 223: 594-597

2. SANKARANARAYANAN, K, DUYN, A, LOOS, M.J. and A.T. NATARAJAN (1989) Adaptive response of human lymphocytes to low-level radiation from radioisotopes and $\mathrm{X}$-rays. Mutation Research, $211: 7-12$

3. VIJAYAXILAMI, G. andW. BURCKART (1989) Resistance and crossresistance to chromosome damage in human blood lymphocytes adapted to bleomycin. Mutation Res. 211: 1-5

4. CORTES, F., DOMINGUEZ, I, MATEOS S.,PINERO, J. and J. C. MATEOS (1990) Evidence for an adaptive response to radiation damage in plant cells conditioned with $\mathrm{X}$-rays or incorporated tritium. Int. I. Radiat. Biol. 57: 537-541

5. CAI, L and S.Z. LIU (1991) Induction of cytogenetic adaptive response in C57 BL mice pre-exposed in vivo and in vitro by low dose Xirradiation.Int. J. Radiat. Biol. 58: 187-194

6. BOSI, A and G. OLIVIRI (1989) Variability of yhe adaptive response to ionising radiations in humans, Mutation Res., 211: 13-17

7. SHADLEY, J.D., AFZAL, V., and S. WOLFF (1987) Characterization of the adaptive response to ionizing radiation induced by low doses of $\mathrm{X}$ rays to human lymphocytes. Radiation Res., $111: 511-517$.

8. WIENCKE, J.K, AFZAL, OLIVIERI, G.and S. WOLFF (1986) Evidence that ${ }^{3} \mathrm{H}$ Thymidine-induced adaptive response of human lymphocytes to subsequent dose of $\mathrm{X}$-rays involves the induction of a chromosomal repair mechanism. Mutagenesis, 1 : 375-380.

9. SANDERSON B. J. S and A. A MORLEY (1986) Exposure of human lymphocytes to ionising radiation reduces mutagenesis by subsequent mutagenesis. Mutation Res. 164: 347-351

10. KELSEY, K. T, MEMISOGLU, A., FRENKEL, D. and H.L. LIBER (1991) Human lymphocytes exposed to low doses of X-rays are less susceptible to radiation induced mutagenesis. Mutat. Res. 263: 197-201

11. RIGAUD, O, PAPADOPOULO, D. and E. MOUSTACCHI (1992) Decreased deletion-type mutation in radiodapted human cells. Radiation Research, 232

12. THACKER, J. (1986) The nature of mutants induced by ionising radiation in cultured cells. III: Molecular characterization of HPRT deficient mutants induced by g-rays or a-particles showing that the majority have deletion of all or part of the HPRT gene. Mutation Res. 160: 267275 